\title{
Calidad y participación en el sistema sanitario en el barrio zaragozano de Casablanca: una reflexión sobre la importancia de la comunidad
}

\author{
Quality and participation in the health care system in the Casablanca \\ district (Zaragoza, Spain): a reflection on the importance of community
}

\author{
Marta GIL LACRUZ \\ Universidad de Zaragoza \\ mglacruz@unizar.es \\ Ana Isabel GIL LACRUZ \\ Universidad de Zaragoza \\ anagil@unizar.es
}

Recibido: 30/01/2012

Revisado: 08/03/2012

Aceptado: 23/04/2012

Disponible on line: 03/08/2012

\begin{abstract}
Resumen
El debate sobre la calidad y sostenibilidad de nuestro sistema sanitario nos remite a cuestiones relativas a la medida de su acceso y valoración por parte de la ciudadanía. La atención primaria centra dichas premisas en el ámbito local y comunitario. En el barrio de Casablanca (Zaragoza) preguntamos a 1.032 vecinos sobre la percepción de sus servicios públicos como recursos potenciales de apoyo y el modo concreto en el que los agentes sanitarios podían mejorar su actuación. Con este fin, se diseñó una encuesta liderada por el personal médico, la trabajadora social del centro de salud y profesores universitarios del ámbito psicosocial. En los resultados del estudio, se constata que variables como el sexo, la edad y la zona de residencia en el barrio influyen tanto en la evaluación de estos recursos sociales como en las alternativas que se proponen para su mejora. El desarrollo de nuestro sistema sanitario requiere no solo de una política anticrisis y de la racionalización presupuestaria, sino también de medidas socioeconómicas que garanticen la equidad en su acceso y la participación comunitaria en su planificación.
\end{abstract}

Palabras clave: salud pública, comunidad, calidad asistencial, participación ciudadana.

\begin{abstract}
The debate over the quality and sustainability of our health care system leads us to questions concerning public access and its value to the public. Primary health care focuses these considerations at the local and community level. In the district of Casablanca (Zaragoza, Spain), 1,032 residents were polled on their perceptions of their health care resources as potential sources of support and the specific way in which health workers could improve their performance. To this end, we designed a survey led by medical staff, the social worker at the local health center and University professors in the psychosocial fields. In the results of the study, we found that variables such as sex, age and area of residence in the neighborhood influence the evaluation of these social resources as well as their alternatives for improvement. The development of our health care system requires not only an anti-crisis policy and budgetary rationalization, but also socio-economic strategies that ensure equity in access and community participation in its planning.
\end{abstract}

Key words: public health, community, health care quality, civic participation.

Referencia normalizada: Gil Lacruz, M., y Gil Lacruz, A.I. (2012): «Calidad y participación en el sistema sanitario en el barrio zaragozano de Casablanca: una reflexión sobre la importancia de la comunidad». Cuadernos de Trabajo Social, 25(2): 345-356.

Sumario: 1. ¿La salud pública como derecho universal? 2. La participación comunitaria como requisito de la salud pública. 3. Diseño metodológico. 4. Resultados. 5. Conclusiones. 6. Referencias bibliográficas. 


\section{1. ¿La salud pública como derecho universal? ${ }^{1}$}

La Constitución española en sus artículos 43 y 49 reconoce el derecho a la salud. La Ley General de Sanidad desarrolla este principio como: el acceso universal a la asistencia sanitaria, la prevención de la enfermedad, la protección y promoción de la salud y el establecimiento de políticas de salud pública que contribuyan a superar las desigualdades sociales y territoriales. Actualmente, en una situación de crisis económica, de envejecimiento de la población, de prevalencia de enfermedades crónicas y estilos de vida perniciosos (por ej. adicciones, estrés, siniestralidad, etc.) y el deterioro del medio ambiente, no se discute la vigencia de este derecho, sino su sostenibilidad, alcance y calidad (Gil-Lacruz, 2011, Mitchell y Schlesinger, 2006).

Ante este panorama, la medicina establece nuevos modelos de práctica basados en la evidencia, que toman en consideración la importancia de las nuevas tecnologías de la información, la conciencia aumentada de riesgo y, como describe Morago (2004): «la consagración de los principios económicos de efectividad, transparencia y atención al consumidor. La práctica basada en la experiencia se ha extendido rápidamente desde el campo de la salud a otras áreas, entre las que se incluye el Trabajo Social» (p. 5). Pese al entusiasmo suscitado, queda pendiente el debate acerca de su «transferibilidad» a un contexto de circunstancias sociales complejas y dispares.

Las dudas y críticas que se vierten sobre nuestro modelo de salud pública nos obligan a la reflexión histórica y al análisis de sus posibilidades en el futuro. Lo cierto es que en nuestro país, a pesar de nuestro retraso democrático, se puede hablar del milagro de la salud pública, puesto que en apenas tres décadas pasamos de ser uno de los países con mayor mortalidad de Europa a ostentar uno de los niveles de salud más elevados (Caïs y Castilla, 1995). Otra cuestión es que esta mejora no haya beneficiado a todos por igual. Las diferencias territoriales, sociales, económicas, etc., se traducen en desigualdades importantes en el acceso a nuestro sistema sanitario (Rodríguez y De Miguel, 1990). Las personas con una situación socioeconómica privilegiada pueden elegir y combinar recursos sanitarios públicos y privados. Este hecho es especialmente relevante en relación a los servicios sanitarios preventivos que no son cubiertos por el sistema público, como por ejemplo determinadas intervenciones dentales y de carácter ortopédico (De Miguel, 1997).

A menudo, nuestro sistema sanitario ignora que los aspectos contextuales y comunitarios influyen en el comportamiento de salud de la población. El abuso de fármacos, de intervenciones tecnologizadas y la medicalización de la población generan no sólo elevados costes económicos para el mantenimiento del sistema, sino también la dependencia de la población (Greaves, 2000). La formación y ejercicio profesional del profesional sanitario, la cultura y expectativas del cliente, el lugar de la consulta, la organización institucional de la salud, etc., nos remiten a la necesidad de considerar los aspectos sociales de nuestra salud (Sofaer y Firminger, 2005).

La salud no sólo implica la evitación o la cura de la enfermedad. Una definición cercana al bienestar supone tanto la satisfacción de las necesidades básicas del individuo y su desarrollo integral, como la adaptación continua a su entorno de referencia (San Martín, 1989). Las desigualdades en los parámetros de morbilidad y en la utilización de los recursos sanitarios son al mismo tiempo causa y consecuencia de desigualdades sociales en función del sexo, la situación socioeconómica, la zona de residencia, etc. (Braveman, 2006; Rooks; 2000).

Además en la valoración de la calidad de nuestro sistema sanitario, la investigación sobre las necesidades comunitarias presenta una serie de alicientes: nos permite identificar a los individuos y los grupos que comparten determinados problemas, facilitando su contextualización en un territorio concreto (McCann, Ryan y McKenna, 2005); nos ayuda a evaluar el impacto de los proyectos comunitarios, aportando información sobre los valores y barreras que reducen el efecto positivo de los programas preventivos (Laporte, Croxford y Coyte, 2007). Como plantean Kenney, Loan y Nicholson (2006), uno de los objetivos más importantes de

\footnotetext{
1 Proyecto de investigación I+D+I Ministerio de Ciencia e Innovación: CSO2011-30089. Comportamiento sexual y reproductivo en España: factores de riesgo para la salud.
} 
los estudios de salud pública estriba en mejorar la comunicación entre los recursos sanitarios y sus clientes.

Estas premisas trascienden el nivel de análisis individual y colocan en la comunidad el protagonismo de la promoción del bienestar (Morton, 2000). Es en la comunidad donde se deberían expresar dichas necesidades compartidas y priorizar su actuación. La participación comunitaria constituye un requisito imprescindible para la definición de nuestra salud como una conquista social compartida. En este sentido, la Declaración de Liverpool sobre el derecho a la salud (Organización Mundial de la Salud, 1988) defendió explícitamente que es la comunidad la que ostenta el derecho y el deber de participar, a nivel colectivo e individual, en los proyectos que afectan a la salud pública, incluida la planificación y gestión de su salud comunitaria y de sus servicios comunitarios.

\section{La participación comunitaria como re- quisito de la salud pública}

La Organización Mundial de la Salud (1981) define dicha participación en los siguientes términos:

El proceso en virtud del cual los individuos y las familias asumen responsabilidades en cuanto a su salud y bienestar propios y los de la colectividad; mejoran la capacidad de contribuir a su propio desarrollo económico y al comunitario, pues al ser los mejores conocedores de su propia situación son capaces de encontrar incentivos que resuelvan sus problemas comunes.

De manera complementaria, también la Carta de Otawa supuso entender como participación la asunción de la diversidad de intereses y grupos sociales en la salud, el cuestionamiento de las leyes del mercado y el establecimiento de una sistema de relaciones comunitarias más equitativo y justo, como objetivos del sistema sanitario (Cornell, 2000).

El desarrollo de la atención primaria y la revalorización de la comunidad en la que se asienta, constituyen la forma de articular en nuestro país la participación comunitaria al defender como metas: la promoción de estilos de vida dirigidos a la salud, la prevención de enfermedades, el establecimiento de servicios de rehabilitación (con especial atención a la humanización de los servicios sanitarios, el diagnóstico precoz, los servicios especiales, la calidad y el cociente costo-efectividad de los servicios, así como la equidad en su provisión). Al entender la salud como un asunto público, es evidente que el gobierno es responsable de fomentar dicha competencia participativa (De Luca, 2000).

La participación comunitaria tiene mucho que ver con el empoderamiento y con los objetivos de la intervención social (Morago, 2006). Presenta dos vertientes complementarias: la del individuo que ejerce el control sobre su propia vida, y la relacionada con la defensa de sus derechos sociales y comunitarios (Musitu y Buelga, 2004). En atención primaria, la coordinación entre instituciones, asociaciones, grupos de autoayuda, plataformas ciudadanas, constituye un canal de participación social al potenciar el sentimiento de pertenencia a la comunidad y ejercer un efecto positivo sobre el bienestar (Hombrados y Gómez, 2001). Ambos niveles se encuentran interrelacionados: tanto la mejora de la gestión de los servicios públicos y condiciones de vida, como la formación de capital social y el desarrollo de una identidad compartida, permiten integrar el concepto de ciudadanía (Pastor, 2008).

La traducción institucional de estas premisas depende en muchos casos de la coyuntura política, económica y de los intereses creados. A menudo, el discurso democrático de la calidad asistencial en el sistema sanitario se hace sólo eco de algo tan básico como es recoger de forma anónima las reclamaciones de sus propios clientes (Sobo, Seid y Reyes, 2006; Tousignant, Duboc, Hebert y Coulombe, 2007).

Las críticas frecuentes a estos instrumentos poblacionales de control apuntan a su tendencia a surgir no tanto por la reivindicación ciudadana, como por el lado de la oferta institucional, corriendo el riesgo de incrementar el gerencialismo y la burocracia por la metodología tan poco participativa que proponen. La misma crítica se puede plantear a la coordinación profesional en el sistema sanitario. A menudo las reuniones de los equipos multidisciplinares tienen como fin fundamental la información y el refrendo de una agenda en la que no hay espacio para la deliberación. A pesar de ello, estructuras como el Consejo de Salud, favorecen la progresiva percepción de «derecho a la salud» frente al concepto de dádiva o asistencialismo y deberían tener un mayor papel prota- 
gónico en la socialización colectiva del desarrollo local, la revitalización del capital social de los municipios, la potenciación de los recursos humanos de los Servicios Sociales de Atención Primaria, el enriquecimiento del debate político y, por lo tanto, de las propias políticas sociales municipales (Pastor, 2008).

Estos fines requieren que la participación ciudadana se analice y desarrolle de forma operativa, colegiada, y en procesos tanto en el derecho a la información (como por ej. campañas preventivas), como en la deliberación y toma de decisiones (por ej. en relación con las políticas sociales). El proceso democratizador debería recoger las preferencias ciudadanas, a la vez que desarrollar estructuras de implicación en las bases asociativas (Pastor, 2008). Como plantea Rubio (2007), se trata de reconocer en la práctica el peso de las organizaciones integrantes de la sociedad civil como Tercer sector, por situarse entre el Estado y el mercado. Este reconocimiento no nos exime de estudiar las diferentes posiciones ideológicas y estrategias interventoras que lo defienden, ni de encarar el ejercicio definitorio de qué es lo que se entiende por su objetivo: el desarrollo comunitario (Casadevante, Haro, Murgui y Ramos, 2006).

En España, además, los estudios de calidad y satisfacción asistencial tienden a arrojar resultados positivos, pudiendo deberse a varios motivos: el reconocimiento poblacional de la labor asistencial, la deseabilidad social de la respuesta, la representación social simplificada de los servicios y el desconocimiento de la labor profesional de sus agentes, el patronazgo institucional del estudio, etc. (Bueno y Pérez, 2000). Las tendencias centrales de satisfacción pueden ocultar importantes diferencias en cuanto al acceso y valoración de dichos recursos (Gil-Lacruz, 2011). La participación es un fin, pero también un medio para acceder y controlar los servicios y solucionar problemas. Con la investigación en el barrio de Casablanca pretendemos analizar la valoración de los recursos sanitarios por parte de la población y su relación con las dinámicas participativas y de integración comunitarias.

\section{Diseño metodológico}

La encuesta de salud de Casablanca (Zaragoza) se desarrolló desde el Centro de Salud del Distrito con una doble finalidad: conocer las pautas epidemiológicas de la población de referencia y analizar los determinantes sociales que influían en su percepción de la salud (Questionnaire on Perception of Health; Davies y Ware, 1981); estilos de vida (Cuestionario sobre Comportamientos de Riesgo para la Salud, Herrero, 1994), apoyo comunitario (Test de Apoyo Comunitario, Gracia y Musitu, 1990), y acceso al sistema sanitario (Encuesta Nacional de Salud, preguntas relativas a morbilidad, número de consultas, motivo y respuesta de la consulta, satisfacción con la consulta). El diseño, implementación y evaluación de este instrumento supuso un trabajo de coordinación multidisciplinar, en el que intervino el equipo médico, de trabajo social y personal experto externo al centro.

Dadas las características socioeconómicas del barrio, Casablanca como comunidad se define por sus contrastes y desigualdades sociales, delimitadas territorialmente en torno a tres grandes zonas:

- Viñedo Viejo, que constituye el núcleo originario del barrio y se caracteriza por presentar una población envejecida, problemas de deterioro de viviendas y de tráfico urbano. Sus residentes se instalaron en esta zona en los años 1960, procedentes en su mayoría del entorno rural aragonés, atraídos por la demanda de mano de obra de la construcción y el desarrollo de Zaragoza capital como polo de desarrollo en el régimen de Franco.

- Las Nieves, que presenta una composición demográfica equilibrada correspondiente a urbanizaciones de clase media. En estos momentos asiste al declive de las empresas y fábricas instaladas en la zona y la emigración de sus residentes más jóvenes por las dificultades de empleo y la especulación urbanística.

- Fuentes Claras, en donde destacan las urbanizaciones de categoría y la presencia de nuevos colonos (por ej. profesionales liberales) que residen en la zona pero se desplazan diariamente a trabajar a otras zonas de Zaragoza capital.

Con el fin de que el estudio fuera representativo en función de la edad, el sexo y la zona de residencia de los vecinos, se entrevistó en su domicilio a 1.032 personas. El tiempo promedio de la entrevista fue de 27 minutos. Las personas 


\begin{tabular}{|l|l|r|r|r|r|r|}
\hline \multirow{3}{*}{ Zona de residencia } & & \multicolumn{2}{|c|}{ Varón } & \multicolumn{2}{|c|}{ Mujer } & \multirow{2}{*}{ Subtotales } \\
\cline { 3 - 7 } & & Recuento & $\begin{array}{c}\text { \% del N } \\
\text { de la zona }\end{array}$ & Recuento & $\begin{array}{c}\text { \% del N } \\
\text { de la zona }\end{array}$ & \\
\hline \multirow{3}{*}{ Viñedo Viejo } & Juventud (< de 30 años) & 70 & $11,3 \%$ & 85 & $13,8 \%$ & 155 \\
\cline { 2 - 7 } & Adultos (de 30 a 59 años) & 152 & $24,6 \%$ & 155 & $25,1 \%$ & 307 \\
\cline { 2 - 7 } & Tercera edad (> de 60 años) & 40 & $6,5 \%$ & 116 & $18,8 \%$ & 156 \\
\hline \multirow{3}{*}{ Las Nieves } & Juventud & 77 & $24,8 \%$ & 53 & $17,1 \%$ & 130 \\
\cline { 2 - 7 } & Adultos & 59 & $19,0 \%$ & 74 & $23,9 \%$ & 133 \\
\cline { 2 - 7 } & Tercera edad & 47 & $15,2 \%$ & 0 & $0,0 \%$ & 47 \\
\hline \multirow{2}{*}{ Fuentes Claras } & Juventud & 26 & $25,0 \%$ & 26 & $25,0 \%$ & 52 \\
\cline { 2 - 7 } & Adultos & 26 & $25,0 \%$ & 26 & $25,0 \%$ & 52 \\
\cline { 2 - 7 } & Tercera edad & 497 & $0,0 \%$ & 0 & $0,0 \%$ & 0 \\
\hline \multirow{2}{*}{ Subtotales } & & & 535 & & 1.032 \\
\hline
\end{tabular}

Tabla 1. Composición de la muestra.

que no respondieron alegaron falta de tiempo, y que algunas cuestiones planteadas en la encuesta, por ej. los ingresos anuales, eran demasiado personales. Las respuestas obtenidas con los instrumentos estandarizados y la transcripción literal de las preguntas abiertas (por ej. en su opinión ¿cuál es la principal fuente de mejora del sistema sanitario?) se codificaron y analizaron, utilizando el software de SPSS.

\section{Resultados}

Un 65 por ciento de los encuestados en el barrio había padecido alguna dolencia o trastorno de salud durante las dos semanas anteriores a la entrevista. No obstante, solo el 30 por ciento de las personas entrevistadas había acudido al médico.

La mayoría de los recursos sanitarios que se han consultado en Casablanca son de titularidad pública: por ej. el centro de salud del barrio $(35,4$ por ciento), otros centros de salud (17 por ciento) y el ambulatorio de especialidades (12,9 por ciento). Con menor frecuencia se encuentra la consulta privada (12,2 por ciento).

\subsection{Fuentes de mejora de los recursos sani- tarios}

A los vecinos encuestados se les pidió que priorizaran cuál creían que era la principal fuente de mejora de sus recursos sanitarios. Las respuestas a esta pregunta abierta se codificaron posteriormente, utilizando el sistema de jueces y pares anónimos. Las principales categorías planteadas hacían referencia a cuestiones relativas a aspectos materiales y de infraestructura, administrativas, comunicativas, técnicas, políticas y relativas a la propia participación ciudadana.

Un 22,5 por ciento de la muestra consideraba que sus recursos sanitarios ya funcionaban adecuadamente. De los que apuntaron posibles fuentes de mejora, un elevado porcentaje de la muestra 46,1 por ciento coincidió en señalar que los servicios sanitarios deberían mejorar en aspectos administrativos, especialmente los relativos a la reducción de las listas de espera. Las políticas sanitarias que se proponían, como por ejemplo, el incremento de las campañas preventivas, mejoras en la planificación y gestión de los recursos, las planteó el 19,7 por ciento de la muestra. En tercer lugar, se encuentran los aspectos comunicativos en la interacción entre los profesionales sanitarios y los clientes $(18,6$ por ciento). Se apuntaron también como temas pendientes: el desarrollo de infraestructura y otras cuestiones materiales (6,6 por ciento) y un mejor acceso a la tecnología médica ( 7,6 por ciento). Solamente el 1,5 


\begin{tabular}{|c|c|c|c|}
\hline \multicolumn{2}{|c|}{$\begin{array}{c}\text { Consultas Casablanca } \\
(\%)\end{array}$} & Lugar de la última visita & $\begin{array}{l}\text { Casablanca } \\
\text { (\% consultas) }\end{array}$ \\
\hline \multirow{11}{*}{$\begin{array}{c}\text { No } \\
69,9 \%\end{array}$} & \multirow{11}{*}{$\begin{array}{c}\text { Sí } \\
30,1 \%\end{array}$} & Centro de Salud de Casablanca (servicios médicos) & $35,4 \%$ \\
\hline & & Centro de Salud de Casablanca (trabajador social) & $3,5 \%$ \\
\hline & & Otros centros de salud & $17,0 \%$ \\
\hline & & Ambulatorio Seguridad Social (especialidades) & $12,9 \%$ \\
\hline & & Servicio urgencias hospital & $4,5 \%$ \\
\hline & & Consulta privada (particular/mutua privada) & $12,2 \%$ \\
\hline & & Empresa o lugar de trabajo & $2,3 \%$ \\
\hline & & Visita del médico de cabecera al domicilio & $0,6 \%$ \\
\hline & & Servicio especial de urgencias a domicilio & $0,3 \%$ \\
\hline & & Consulta telefónica & $0,0 \%$ \\
\hline & & Consulta externa en hospital & $8,0 \%$ \\
\hline
\end{tabular}

Tabla 2. Porcentaje y lugar en el que se efectúan las consultas médicas realizadas en Casablanca.

por ciento de la muestra expresó que los ciudadanos tienen cierto protagonismo en dichas mejoras.
Las alternativas propuestas para la mejora del sistema sanitario dependen de manera estadísticamente significativa, entre otros de los si-

\begin{tabular}{|c|c|c|c|c|}
\hline Categorías & Respuestas literales & $\begin{array}{c}\% \\
\text { hombre }\end{array}$ & $\begin{array}{c}\% \\
\text { mujer }\end{array}$ & $\begin{array}{l}\% \text { total } \\
\text { muestra }\end{array}$ \\
\hline $\begin{array}{l}\text { Material } \\
\text { e infraestructura }\end{array}$ & $\begin{array}{l}\text { «El dinero público», «más hospitales», } \\
\text { «más consultorios», «poner más servicios», } \\
\text { «más medios» }\end{array}$ & $6,9 \%$ & $6,2 \%$ & $6,6 \%$ \\
\hline $\begin{array}{l}\text { Cuestiones } \\
\text { Administrativas }\end{array}$ & $\begin{array}{l}\text { «Menos listas de espera en especialistas», } \\
\text { «más rápida la atención» }\end{array}$ & $45,6 \%$ & $46,4 \%$ & $46,1 \%$ \\
\hline Comunicación & $\begin{array}{l}\text { «Asistencia más humana», «escuchar a la } \\
\text { gente», «personal más amable», «atención } \\
\text { más personalizada» }\end{array}$ & $15,9 \%$ & $21,3 \%$ & $18,6 \%$ \\
\hline Cuestiones técnicas & $\begin{array}{l}\text { «Más profesionalidad», «médicos más } \\
\text { competentes», «mejor competencia», «que } \\
\text { haya buenos profesionales» }\end{array}$ & $5,1 \%$ & $10,1 \%$ & $7,6 \%$ \\
\hline Política sanitaria & $\begin{array}{l}\text { «Mejor planificación», «privatización», } \\
\text { «llevar a cabo el informe Abril Martorell». }\end{array}$ & $24,3 \%$ & $15,1 \%$ & $19,7 \%$ \\
\hline Propios usuarios & $\begin{array}{l}\text { «Mayor participación», «colaboración de } \\
\text { todos», «más paciencia de los usuarios», } \\
\text { «utilizar correctamente los servicios» }\end{array}$ & $2,1 \%$ & $0,9 \%$ & $1,5 \%$ \\
\hline \multicolumn{2}{|l|}{ Totales } & $100 \%$ & $100 \%$ & $100 \%$ \\
\hline
\end{tabular}

Tabla 3. Categorías temáticas utilizadas en la sistematización de las respuestas a la principal fuente de mejora de los servicios de salud. 
guientes factores: el sexo, la edad, la zona de residencia y el estado de salud del encuestado.

\subsubsection{Sexo y edad}

Encontramos un mayor porcentaje de respuesta específica, a la pregunta de si es posible mejorar el sistema sanitario, entre los varones que entre las mujeres residentes en el barrio, seguramente debido a que entre las encuestadas se da una mayor frecuencia de respuesta relativa a que el sistema se considera que funciona adecuadamente (un 25,4 por ciento de las entrevistadas está satisfecha con los servicios sanitarios, frente a un 19,3 por ciento de los hombres) $\left(\chi^{2}=6,05157 ; \mathrm{gl}=2 ; \mathrm{p}=0,04852<\mathrm{p}=0,05\right)$.

En relación a qué aspectos se podrían mejorar, los varones priorizaron las cuestiones materiales o de infraestructura, la política sanitaria y el papel de los propios usuarios. En cambio, las mujeres se decantaron más por las cuestiones administrativas (por ej. listas de espera), la relación entre médico paciente y los aspectos técnicos de dichos servicios $\left(\chi^{2}=17,10836\right.$; $\mathrm{gl}=5 ; \mathrm{p}=0,00430<\mathrm{p}=0,01)$.

Se constata una tendencia similar en relación con la variable edad (respuesta a la mejora del sistema sanitario $\chi^{2}=113,56827 ; \mathrm{gl}=4$; $p=0,00000<p=0,01)$. Los jóvenes apuntaron un mayor número de alternativas al funcionamiento sanitario $(73,9$ por ciento) que los encuestados mayores de 60 años $(41,9$ por ciento), posiblemente porque en este último colectivo se ha constatado un mayor número de respuestas relativas a que el sistema no necesita mejorar $(47,3$ por ciento de las personas de edad avanzada plantearon que los servicios cumplían su misión adecuadamente, frente al 8,9 por ciento de los menores que respondieron de esta manera) $\left(\chi^{2}=113,56827 ; \mathrm{gl}=4 ; \mathrm{p}=0,00000<\right.$ $\mathrm{p}=0,01)$.

Si la comparación intragrupos la realizamos en función de la edad, observamos que los jóvenes de la muestra encuestada destacaron en el porcentaje de respuestas referentes al trato humano y a la política sanitaria. Los adultos aludieron con más frecuencia a las cuestiones administrativas. En los encuestados mayores de 60 años han predominado las respuestas relativas a: los factores materiales y de infraestructura, las cuestiones técnicas y el papel de los usuarios en dichas mejoras $\left(\chi^{2}=21,47197 ; \mathrm{gl}=10\right.$; $\mathrm{p}=0,01803<\mathrm{p}=0,05)$.

\subsubsection{Zona de residencia}

Los vecinos residentes en la zona de nueva colonización (Fuentes Claras) y en general con un estatus elevado, plantearon con más frecuencia que no sabían o no contestaron cómo mejorarían los servicios sanitarios $(25,0$ por ciento de los residentes en el área, frente al 10,7 por ciento de los residentes en Viñedo Viejo) y al mismo tiempo, representan el porcentaje de respuesta favorable al funcionamiento actual más bajo $(5,8$ por ciento), frente a los vecinos del núcleo originario $\left(30,4\right.$ por ciento) $\left(\chi^{2}=68,27006 ; \mathrm{gl}=4\right.$; $\mathrm{p}=0,00000<\mathrm{p}=0,01)$.

El porcentaje de respuestas favorables al sistema y el de alternativas de mejora, está asociado con los años de residencia en el barrio: a mayor tiempo de asentamiento (especialmente en el colectivo que lleva viviendo más de cuatro décadas en Casablanca) se apunta más satisfacción con el sistema y menos opciones de cambio $\left(\chi^{2}=67,56833 ; \mathrm{gl}=8 ; \mathrm{p}=0,00000<\right.$ $\mathrm{p}=0,01)$. Los nuevos colonos se centraron en la política sanitaria, el papel de los propios usuarios y un trato más humano. Las personas que llevaban residiendo en Casablanca de 6 a 19 años, preferirían ciertas mejoras relativas a los aspectos administrativos y técnicos. Los vecinos con más antigüedad apuntaron hacia la conveniencia de que los servicios sanitarios estuvieran mejor dotados a un nivel material, de infraestructura y técnico $\left(\chi^{2}=33,89760 ; \mathrm{g}=20\right.$; $\mathrm{p}=0,02683<\mathrm{p}=0,05)$.

\subsubsection{Pautas epidemiológicas}

El colectivo encuestado que ha padecido alguna dolencia durante los últimos 15 días o cuyo grado de incapacidad es medio-severo, aportan más respuestas y está menos satisfecho con los servicios sanitarios que el colectivo sin dolencias o con un menor grado de incapacidad. Sin embargo, aquellos que han padecido dolencias crónicas, se sitúan en la posición contraria, siendo los que plantean con mayor frecuencia que los servicios sanitarios no requieren mejora. Las personas que no han padecido ninguna incapacidad, como resultado de sus problemas de salud, son quienes menos respuestas expresan acerca de la mejora del sistema sanitario. (WONCA diagnóstico médico $\chi^{2}=22,71143$; $\mathrm{gl}=6 ; \mathrm{p}=0,00090<\mathrm{p}=0,01)$ (morbilidad aguda $\left.\chi^{2}=15,65643 ; \mathrm{gl}=2 ; \mathrm{p}=0,00040<\mathrm{p}=0,01\right)$ (morbilidad crónica $\chi^{2}=15,17793 ; \mathrm{gl}=2 ; \mathrm{p}=$ 
$0,00051<\mathrm{p}=0,01)\left(\right.$ incapacidad $\chi^{2}=18,58237$; $\mathrm{gl}=8 ; \mathrm{p}=0,01726<\mathrm{p}=0,05)$.

Los vecinos de Casablanca que han sufrido alguna enfermedad o dolencia, que ha durado más de tres meses de forma permanente, exponen que dichos servicios deberían mejorar en cuanto a su dotación material, de infraestructura, y otras cuestiones técnicas o relativas a la política sanitaria. Los encuestados, que podríamos calificar de sanos, hacen hincapié en las cuestiones administrativas, las relaciones humanas o su propio papel en la mejora de dichos servicios (morbilidad crónica $\chi^{2}=18,00273$; $\mathrm{gl}=5 ; \mathrm{p}=0,00294<\mathrm{p}=0,01)$.

\subsection{Procesos de integración y participación comunitaria}

Con el fin de medir el grado de integración y participación comunitaria, se utilizó el cuestionario de Apoyo Comunitario de Gracia y $\mathrm{Mu}-$ situ (1990). Esta herramienta mide también el grado en que las personas de un barrio confían en sus recursos gubernamentales y no gubernamentales como fuentes potenciales de apoyo en caso de necesidad.
En Casablanca, se aprecian resultados diferentes en la percepción del apoyo proveniente de los sistemas formales, siendo las mujeres más proclives a una valoración positiva que los varones $(F=11,0314 ; p=0,009) \quad(13,3481<$ 13,6710) $(\alpha=0,05)$. También este hecho, se constata en los resultados obtenidos en la investigación de Herrero (1994).

La participación $(\mathrm{F}=22,6627 ; \mathrm{p}<0,0001)$ $(16,0742<16,7663<17,5320)(\alpha=0,05)$ y la integración comunitaria $(\mathrm{F}=46,6635 ; \mathrm{p}<0,0001)$ $(14,1632<15,0122<15,7241) \quad(\alpha=0,05)$, están relacionadas con la edad de los encuestados, siendo las personas mayores las que presentaron una puntuación mayor, seguida de los adultos, encontrándose diferencias significativas entre los tres grupos.

Estas diferencias también parecen traducirse en lo que respecta a la percepción de apoyo proveniente tanto de los sistemas formales $(\mathrm{F}=$ $19,1709 ; \mathrm{p}<0,0001)(13,1454<13,5793<13,9754)$ $(\alpha=0,05)$ como de los informales $(\mathrm{F}=12,0252$; $\mathrm{p}<0,0001)(30,3650<30,7602<31,5567)(\alpha=$ $0,05)$, encontrándose como matiz para este último sistema el hecho de que no se observaron

\begin{tabular}{|l|r|r|r|c|c|}
\hline & SC & F & P & Hombres & Mujeres \\
\hline Sexo & & & & & \\
Participación & 0,0442 & 0,0070 & 0,9334 & 16,6841 & 16,6972 \\
Integración & 8,6193 & 2,2669 & 0,1325 & 14,9698 & 14,7869 \\
Sistemas informales & 0,1624 & 0,0211 & 0,8844 & 30,8008 & 30,7757 \\
Sistemas formales & 26,8703 & 11,0314 & $\mathbf{0 , 0 0 0 9}$ & 13,3481 & 13,6710 \\
\hline & gl & SC & MC & F & P \\
\hline Edad & & & & & \\
Participación & 2 & 274,5872 & 137,2936 & 22,6627 & $\mathbf{0 , 0 0 0 0}$ \\
Integración & 2 & 326,3727 & 163,1864 & 46,6635 & $\mathbf{0 , 0 0 0 0}$ \\
Sistemas informales & 2 & 180,6216 & 90,3108 & 12,0252 & $\mathbf{0 , 0 0 0 0}$ \\
Sistemas formales & 2 & 91,0912 & 45,5456 & 19,1709 & $\mathbf{0 , 0 0 0 0}$ \\
\hline Zona de residencia & & & & & \\
Participación & 2 & 2,2892 & 1,1446 & 0,1810 & 0,8344 \\
Integración & 2 & 113,8645 & 56,9323 & 15,3721 & $\mathbf{0 , 0 0 0 0}$ \\
Sistemas informales & 2 & 0,8332 & 0,4166 & 0,0542 & 0,9472 \\
Sistemas formales & 2 & 12,7450 & 6,3725 & 2,5990 & 0,0748 \\
\hline Años de residencia & & & & & \\
Participación & 2 & 65,4108 & 32,7054 & 5,2233 & $\mathbf{0 , 0 0 5 5}$ \\
Integración & 2 & 117,5529 & 58,7765 & 15,8854 & $\mathbf{0 , 0 0 0 0}$ \\
Sistemas informales & 2 & 42,0601 & 21,0301 & 2,7509 & 0,0643 \\
Sistemas formales & 2 & 58,5864 & 29,2932 & 2,1682 & $\mathbf{0 , 0 0 0 0}$ \\
\hline
\end{tabular}

Tabla 4. Análisis de varianza para la explicación de la variable apoyo comunitario. 
diferencias significativas entre las personas jóvenes y los adultos.

No se observan diferencias estadísticamente significativas entre las zonas de residencia en cuanto a la participación comunitaria ni en la confianza depositada en los distintos recursos sociales susceptibles de prestar ayuda al vecindario. No obstante, se detectan percepciones diferenciales en cuanto a la identificación con el barrio. La zona de reciente colonización (Fuentes Claras) es la que menos se integra en la dinámica comunitaria, si se comparan los resultados obtenidos con Viñedo Viejo y las Nieves. $(F=15,3721 ; p<0,0001)(14,9709<15,0161$ $>13,8846)(\alpha=0,05)$.

Los vecinos que han participado más en la vida comunitaria del distrito son aquellos que residen en el mismo desde hace 20 años o más (contrastando los resultados obtenidos en este colectivo con los que llevan instalados en $\mathrm{Ca}$ sablanca entre 6 y 19 años $)(F=5,2233 ; p=$ $0,0055)(16,4022<16,9397)(\alpha=0,05)$. También son los encuestados que han convivido durante más años en el barrio, quienes manifestaron sentirse más integrados y valoraron de forma más positiva el posible apoyo proveniente de los sistemas formales del barrio (Integración: $\mathrm{F}=15,8854 ; \mathrm{p}<0,0001)(14,4375<14,5894$ $<15,2101)(\alpha=0,05)$; (Sistemas formales: $F=$ $12,1682 ; \mathrm{p}<0,0001) \quad(13,2188<13,3073<$ $13,7529)(\alpha=0,05)$.

\subsection{Interacción de las fuentes de mejora de los recursos sanitarios con la participación comunitaria}

Pese a que los vecinos de Casablanca comparten una visión positiva de las organizaciones de su barrio, expresan que, en caso de necesidad de apoyo, acudirían con mayor frecuencia a asociaciones no gubernamentales (por ej. de vecinos, culturales, deportivas, cívicas, etc.) que a instituciones como los Servicios Sociales, los centros educativos o los centros de salud.

La interpretación de las variables de apoyo comunitario presenta una tendencia común en las respuestas referentes a los comportamientos sanitarios. El nivel de satisfacción con los servicios sanitarios está relacionado con una postura activa en el barrio. Los sujetos que no participan y que no confían en los recursos formales de la comunidad (como posibles fuentes de solidaridad), son los que comparten una per- cepción más negativa de los servicios sanitarios.

De este modo, cuanto más activa es la participación comunitaria de los entrevistados mayor es la satisfacción con los servicios sanitarios (Correlación: $-0,1225 ; \mathrm{p}=0,031<0,05)$. Y a mayor confianza en los sistemas formales, como recursos de ayuda en caso de necesidad, mayor satisfacción con la consulta sanitaria (Correlación: $-0,1873 ; p=0,001<0,01)$. De manera complementaria, las personas que consideran los servicios institucionales como posibles fuentes de apoyo están más satisfechos con las consultas médicas (ANOVA: $F=8,6402 \mathrm{p}=$ 0,0035 ) (alta confianza $1,5606<$ baja confianza 2,0000) $(\alpha=0,01),\left(\chi^{2}=10,37705 ; \mathrm{gl}=2\right.$; $\mathrm{p}=0,00558<\mathrm{p}=0,01)$.

La integración, la participación comunitaria y la confianza en los sistemas formales explican parte de la representación social de las posibles mejoras del sistema sanitario. El colectivo de personas que está menos integrado en el barrio corresponde al que más alternativas de respuesta apunta en relación con los servicios comunitarios y el que con mayor frecuencia se sitúa en la categoría «no sabe, no contesta». Uno de cada cuatro encuestados que confían en los sistemas institucionales responde que el sistema sanitario ya funciona adecuadamente y no necesita mejorar. La desconfianza en estos servicios se asocia a las alternativas específicas y también a la ausencia de la respuesta. Asimismo los sujetos, que con mayor frecuencia adoptan una postura de participación activa en el barrio, están más satisfechos con los agentes sanitarios de la comunidad, y aquellos que podríamos calificar de menos activos simultanean un mayor absentismo en esta contestación con más respuestas específicas: (integración $\left.\chi^{2}=14,82432 ; \mathrm{gl}=2 ; \mathrm{p}=0,00060<\mathrm{p}=0,05\right)$ (participación $\chi^{2}=6,70450 ; \mathrm{gl}=2 ; \mathrm{p}=0,03501$ $<\mathrm{p}=0,05)$ (sistemas informales $\chi^{2}=4,49308$; $\mathrm{gl}=2 ; \mathrm{p}=0,01576>\mathrm{p}=0,05)$ (sistemas formales $\chi^{2}=15,63837 ; \mathrm{gl}=2 ; \mathrm{p}=0,00040<\mathrm{p}=$ $0,05)$.

\section{Conclusiones}

El primer dato que llama la atención de los resultados obtenidos en Casablanca es que, contrariamente a la idea que se comparte sobre cómo la población tiende a utilizar en exceso los recursos sanitarios, se observa en el barrio que 
solo la mitad de las personas entrevistadas que padecían una dolencia habían acudido a dichos servicios.

En la evaluación de la mejora de los servicios sanitarios aportada por los usuarios, encontramos elementos que invitan a la reflexión sobre nuestro modelo de atención sanitaria. Las listas de espera, el exceso de burocracia, la derivación o la «deriva» de los clientes, son elementos comunes en las respuestas. En relación a la comunicación entre el sistema sanitario y el cliente se critica el reducido tiempo asignado a la consulta, la escasa información que los pacientes reciben y la jerga utilizada que hace difícil la comprensión del diagnóstico y el tratamiento.

Nuestros resultados muestran que la calidad percibida de los recursos sanitarios depende de la interacción de los factores sociales con los epidemiológicos y los comunitarios. En relación a la influencia de la variable sexo, autores como Humphrey (2007) recomiendan que se enmarquen las desigualdades de acceso al sistema sanitario por parte de las mujeres como un factor más amplio de estructuración social $\mathrm{y}$ de violencia de género. Como en otras investigaciones, las variables demográficas y socioeconómicas se encuentran interrelacionadas, por ejemplo en relación al sexo y el nivel ocupacional (Messing y Stellman, 2006). El territorio, en este caso la zona de residencia en el barrio, constituye un reflejo evidente de como las variables de estructuración social se pueden mapear.

En cuanto a los factores epidemiológicos, no es fácil discernir en nuestro estudio hasta qué punto las respuestas de los vecinos son relativas a su padecimiento de trastornos y, por lo tanto, a una mayor interacción con los recursos sanitarios; o si estas variables dependen a su vez de la edad, el sexo o la situación socioeconómica del encuestado, que claramente condicionan el nivel de salud y de enfermedad. Nuevas líneas de investigación nos llevarían a contemplar la posible delimitación de este impacto.

La valoración de los encuestados sobre las alternativas de mejora del sistema sanitario apunta a un déficit en la participación institucional. Los usuarios de los recursos sanitarios del barrio consideran que la responsabilidad para estos progresos, la ostenta fundamentalmente el personal médico y no los políticos, la Adminis- tración Pública o los propios grupos de consumidores y usuarios. Este dato nos remite al importante papel de la educación en la salud y el empoderamiento comunitario que deberían manejar estos profesionales (Allen, Lehner, Mattison, Miles y Russell, 2007). La medicalización de nuestro sistema sanitario nos lleva a la indefensión y a actitudes cívicas cercanas a la pasividad.

¿En qué grado los recursos sanitarios se ajustan a las necesidades y son fuentes potenciales de bienestar del usuario? En la respuesta a esta cuestión la valoración de la población es importante: si se mejoran los niveles de satisfacción de los clientes, se mejora también la calidad de la atención dispensada y se fomenta la participación comunitaria. En nuestra muestra es relevante el hecho de que la confianza en los recursos comunitarios, sean o no de titularidad pública, se predice mutuamente; es decir, las personas que valoran las asociaciones de su entorno tienden a percibir también sus servicios gubernamentales como fuentes potenciales de ayuda.

Como interventores sociales en salud, la interacción del individuo con la comunidad sigue siendo nuestra asignatura pendiente (Markova, 2000). La participación comunitaria integra diversas cuestiones psicosociales como el estudio de las necesidades sociales, las representaciones sociales, sus identidades y el correspondiente reparto de poder.

Participar en la mejora de nuestras comunidades y de nuestro sistema sanitario también nos permite desarrollar nuestra capacidad para luchar contra las desigualdades y mejorar nuestra salud como bienestar biopsicosocial. Este objetivo requiere su aterrizaje en nuestro territorio y en las instituciones de referencia. El Centro de Salud y el Consejo de Salud pueden erigirse como interlocutores entre las políticas sanitarias y su comunidad, diseñando estrategias de comunicación y participación (López y Silas, 1993).

La toma de conciencia, la participación y el cambio constituyen una triada indisoluble, pero de difícil consecución en colectivos desprotegidos (Jiménez y Gil-Lacruz, 2007). La equidad y la justicia distributiva constituyen una constante de las definiciones de bienestar en su doble vertiente individual y social (Jaraíz, 2012). Las políticas públicas pueden mejorar nuestro 
bienestar, legislando y actuando sobre los factores y determinantes sociales que generan desigualdad. Su objetivo es facilitar las condiciones sociales bajo las cuales las personas pueden disfrutar de las mismas oportunidades para desarrollar su calidad de vida (Gil-Lacruz, 2007; Guiteras, 2012; López, 2003).

El actual cuestionamiento de nuestro sistema de salud requiere tanto un marco de análisis global, internacional, socioeconómico y epidemiológico como un enfoque micro, basado en la solidaridad y el reparto, que analice y actúe sobre las causas sociales del enfermar y del ac- ceso desigual al bienestar. Para reducir las desigualdades de salud, no solo debe implicarse el sector sanitario, facilitando el estudio y la igualdad de acceso a dichos servicios, sino que se deben reorientar también las políticas sociales dirigidas a reducir las desigualdades en las condiciones locales de vida (Gil-Lacruz y Gil-Lacruz, 2009). Nuestro rol investigador e interventor se puede plasmar en estrategias cuya finalidad consista en minimizar el impacto de dichas desigualdades desde la esfera privada (por ej. la vivienda) a la pública (el vecindario, los sistemas públicos) (Juan, 2010).

\section{Referencias bibliográficas}

Allen, N.E., Lehner, A., Mattison, E., Miles, T. y Russell, A. (2007). Promoting systems change in the health care response to domestic violence. Journal of Community Psychology, 35, 103-120.

Braveman, P. (2006). Health disparities and health equity. Concepts and measurement. Annual Review of Public Health, 27, 167-194.

Bueno, J. y Pérez, J.V. (2000). Percepción de los servicios sociales y representaciones de los trabajadores sociales. Cuadernos de Trabajo Social, 13, 53-74.

Caïs, J. y Castilla, E.J. (1995). El sector sanitario. En Fundación Foessa (Ed.), V Informe Sociológico sobre la situación social en España. Síntesis. Documentación Social, 101, 227-272.

Casadevante, J.L.F., Haro, J., Murgui, I. y Ramos, A. (2006). ¿Desarrollo comunitario? No lo sé, dímelo tú... (interrogantes desde una iniciativa vecinal). Cuadernos de Trabajo Social, 19, 347-365.

Cornell, R.W. (2000). Charting futures for sociology: sociology and world market society. Contemporary Sociology, 29, 291-296.

Davies, A. y Ware, C. (1981). Conceptualizations and measurement of health and patients for adults in the Health Insurance Study. En J. Herrero (1994). Estresores sociales y recursos sociales: el papel del apoyo social en el ajuste bio-psico-social. Valencia: Tesis doctoral. Facultad de Psicología. Universidad de Valencia.

DeLuca, M.A. (2000). Health reform in public systems: recent reforms in the U.K's National Health Service and the U.S. Veterans Health Administration. The Humanities and Social Sciences, 60, 11 (4202-A).

De Miguel, A. (1997). La sociedad española, 1996-1997. Madrid: Complutense.

Gil-Lacruz, M. (2007). Psicología social y bienestar. Zaragoza: Prensas Universitarias.

Gil-Lacruz, M. (2011). El laberinto de la salud pública. Barcelona: Erasmus

Gil-Lacruz, A.I. y Gil-Lacruz, M. (2009). Dinámicas comunitarias y acceso al sistema sanitario: una participación desigual. Revista Internacional de Organizaciones, 2, 109-130.

Gracia, E. y Musitu, G. (1990). Integración y participación en la comunidad. Una conceptuación empírica del apoyo social comunitario. En G. Musitu, E. Berjano y J.R. Bueno (Eds.). Psicología Comunitaria. Valencia: Nau Llibres.

Greaves, D. (2000). The creation of partial patients. Cambridge Quarterly of Healthcare Ethics, 9, 23-37.

Guiteras, A. (2012). La relación entre lo público y privado en los Servicios Sociales: el papel del tercer sector. Cuadernos de Trabajo Social, 25, 125-132.

Herrero, J. (1994). Estressores sociales y recursos sociales el papel del apoyo social en el ajuste bio-psico-social. Tesis doctoral. Valencia: Facultad de Psicología. Universidad de Valencia.

Hombrados, M.I. y Gómez, L. (2001). Potenciación en la intervención comunitaria. Intervención psicosocial, 10 (1), 56-69.

Humphrey, C. (2007). A Health inequalities perspective on violence against women. Health and Social Care in the Community, 15, 120-127. 
Jaraíz, G. (2012). La dimensión comunitaria en Servicios Sociales. Una aproximación a su tratamiento en barrios vulnerables. Cuadernos de Trabajo Social, 25, 113-124.

Jiménez, T. y Gil-Lacruz, M. (2007). Psicología comunitaria y plataformas ciudadanas: el reto del bienestar. Revista Iberoamericana de Autogestión y Acción Comunal, 50, 41-58.

Juan Toset, E.M. (2010). Aportaciones del trabajo social al análisis de la dimensión territorial en los procesos de exclusión. Una aproximación al ejercicio profesional en el ámbito residencial. Cuadernos de Trabajo Social, 23, 125-144.

Kenney, G., Loan, J. y Nichols, L. (2006). Toward a more reliable federal survey for tracking health insurance coverage and access. Health Services Research, 41, 918-945.

Laporte, A., Croxford, R. y Coyte, P.C. (2007). Can publicly funded home care system successfully allocate service based on perceived need rather than socioeconomic statuts? A Canadian experience. Health and Social Care in the Community, 15, 108-119.

López-Fernández, L.A. y Silas, D. (marzo, 1993). La participación comunitaria. Verdades y Falacias. Revista Centro de Salud, 103.

López Luis, S. (2003). ¿Se puede hablar de calidad de vida en el contexto del Trabajo Social? Cuadernos de Trabajo Social, 16, 109-119.

Markova, I. (2000). Psychology and social change. Londres: London School Seminar Series.

McCann, S. Ryan, A.A. y McKenna, H. (2005). The challenges associated with providing community care for people with complex needs in rural areas: a qualitative investigation. Health and Social Care in the Community, 13, 462-469.

Messing, K. y Stellman, J.M. (2006). Sex, gender and women's occupational health: The importance of considering mechanism. Environmental Research, 101, 149-162.

Mitchell, S. y Schlesinger, M. (2006). Managed care and gender disparities in problematic health care experiences. Health Services Research, 101, 149- 162.

Morago, P. (2004). Práctica basada en la evidencia de la Medicina al Trabajo Social. Cuadernos de Trabajo Social, 17, 5-20.

Morago, P. (2006). Tratamiento comunitario asertivo: práctica, consideraciones éticas y rol del trabajador social. Cuadernos de Trabajo Social, 19, 7-23.

Morton, L.W. (2000). Community perspectives on rural health. Association Paper. American Sociological Association.

Musitu, G. y Buelga, S. (2004). Desarrollo comunitario y potenciación. En G. Musitu, J. Herrero, L.M. Cantera y M. Montenegro (dirs.). Introducción a la psicología comunitaria (pp.167-193). Barcelona: UOC.

Organización Mundial de la Salud (1981). Global strategy for health for all by the year 2000. Ginebra: WHO.

Pastor, E. (2008). Mecanismos de participación ciudadana en los servicios sociales municipales. Las realidades de la Región de Murcia. Cuadernos de Trabajo Social, 21, 7-25.

Rooks, R.N. (2000). Racial differences in self-assessed health: biological or social? Association Paper. American Sociological Association.

Rubio, J.A. (2007). El tercer sector frente a las transformaciones del Estado de Bienestar. Cuadernos de Trabajo Social, 20, 275-287.

San Martín, H. (1989). Manual de Salud Pública y Medicina Preventiva. Barcelona: Masson.

Sobo, E.J., Seid, M. y Reyes, I. (2006). Parent identified barriers to paediatric health care: a process oriented model. Health Services Research, 41, 148 - 172.

Sofaer, S. y Firminger, K. (2005). Patients perceptions of the quality of health services. Annual Review of Public Health, 26, 513-524.

Tousignant, M., Dubuc, N., Hebert, R. y Coulombe, C. (2007). Home-care programmes for older adults with disabilities in Canada: How can we assess the adequacy of services provided compared with the needs of users? Health and Social Care in the Community, 15, 108-119. 